\title{
Prognostic value of carotid ultrasound lesion morphology in retinal ischaemia: result of a long term follow up
}

\author{
C M O'Farrell, D E FitzGerald
}

\begin{abstract}
The importance of carotid plaque morphology in the prognosis of retinal ischaemia was investigated in a group of 165 patients followed for 2-7 years (mean 3.3 years). All patients had an initial carotid duplex ultrasound examination, with the results expressed in terms of the degree of stenosis caused by the lesion, and the lesion morphology. Lesions were divided into two groups, (a) combined homogeneous and simple heterogeneous structures, and (b) complex heterogeneous plaques. Complex heterogeneous plaques had a low echo pool within the lesion and/or an irregular surface pattern. A total of $144(87 \%)$ patients were successfully followed, and of these 37 (26\%) had cerebrovascular, cardiovascular, or retinal ischaemic events in the follow up period; $14(10 \%)$ cerebrovascular accidents (eight fatal), 17 (12\%) myocardial infarctions (10 fatal), two episodes of amaurosis fugax, and one of tunnel vision caused by a retinal embolus were recorded. There was no report of subsequent blindness. The percentage stenosis caused by the carotid lesions, although more severe in the vascular event group, was not significantly different between the groups. However, a significant difference $(p<0 \cdot 1)$ was found in the morphological characteristics of the carotid lesions between the groups. Patients who suffered a vascular event in the follow up period had significantly more complex heterogeneous lesions compared with simple heterogeneous/homogeneous lesions, than those patients who remained alive and well. Carotid endarterectomy and antiplatelet therapy were equally distributed between the event and non-event groups. This suggests that the criteria for selection for treatment should be based on the leșion morphology as well as the degree of stenosis.

(Br f Ophthalmol 1993; 77: 781-784)
\end{abstract}

Carotid arterial disease has been shown to be a significant factor in the development of retinal ischaemic symptoms. ${ }^{1-3}$ The role of severe carotid stenosis as a cause of retinal ischaemia is accepted. ${ }^{47}$ The morphological characteristics of the atherosclerotic lesion as demonstrated by duplex ultrasound is also important. It has been shown that particular types of morphological appearances are linked to a higher incidence of clinical symptoms, even in lesions that hitherto might have been considered relatively insignificant in terms of the amount of stenosis caused by them. ${ }^{12}$ As a result of this finding, it is important to investigate the relationship between carotid lesion morphology, as seen on ultrasound image, and the long term clinical outcome for the patient. This report gives the follow up findings of 165 patients with retinal ischaemia, ${ }^{1}$ to determine whether patients most at risk from further clinical events can be identified by ultrasound evaluation of the carotid arteries.

\section{Materials and methods}

A total of 165 patients (101 men, 64 women: mean age 58 years), who presented with symptoms of retinal ischaemia, were referred from an ophthalmological centre for duplex examination of the extracranial carotid system.

All the examinations were performed by two experienced vascular ultrasonographers, using an ATL MK5 system with a $5 \mathrm{MHz}$ B-mode combined with a $7.5 \mathrm{MHz}$ Doppler transducer. The common, internal, and external carotid arteries were examined for evidence of atherosclerotic lesions or disturbance in blood flow patterns.

The presence or absence of atherosclerotic lesions was established, and where disease was found the degree of stenosis caused was noted together with a description of the ultrasound plaque appearance of the lesion. Morphology was classified as being either (a) homogeneous, with an even echo pattern throughout the lesion, or (b) heterogeneous, with a mixture of low level and high level echoes within the lesion. Heterogeneous plaques were further subdivided into simple and complex groups. Simple heterogeneous plaques had a smooth surface and a more or less even distribution of low and high level echoes; complex structures displayed low echo areas within the lesion and may also have had an irregular or pitted surface at the interface to flowing blood.

Complex heterogeneous lesions, which are associated with symptoms, were compared with simple heterogeneous lesions combined with homogeneous lesions, which are not associated with symptoms, as previously observed. ${ }^{1}$

The patients were followed up over a period of 2-7 years (mean 3.3 years) after the initial duplex ultrasound examination. The hospital records of each patient were examined, and any cerebrovascular, cardiovascular, or retinal ischaemic symptoms suffered by the patient since the date of the ultrasound examination were documented. The current medical condition of the patient was also recorded. In those patients not currently attending the hospital, the information was obtained from the family doctor. In the event of death, the cause was recorded. Patients still alive were separated into those who had 
suffered from subsequent clinical events and those who were without subsequent events from the time of the initial examination. Note was made of any surgical or medical treatment received by the patient since that time.

A comparison was made between those patients who suffered a vascular event during the follow up period and those who remained alive and well throughout.

\section{Statistical analysis}

The $\chi^{2}$ method of statistical analysis was used throughout.

\section{Results}

Follow up information was successfully obtained on 144 patients $(87 \%)$; 21 were lost to follow up.

There were $32(22 \%)$ recorded mortalities, and of those the cause of death was 18 from cerebrovascular or cardiovascular events, 10 malignancy, one pneumonia, and in three the exact cause could not be ascertained.

Of the 122 patients surviving at the time of follow up, 90 reported no subsequent events. In the remaining 22 patients, 19 suffered vascular or retinal events and three non-vascular ophthalmic events.

Duplex ultrasound examination showed carotid artery disease in $30(81 \%)$ patients who reported subsequent vascular events, and 66 (73\%) patients who remained event free during the follow up period. A total occlusion of the carotid artery was found in five (13\%) patients in the former group and three (3\%) patients in the event free group. Where there is total occlusion of the carotid artery, the appearance of the contents of the lesion can be evaluated, but comment of the surface characteristics is not possible. For this reason, the eight patients with occlusion of the carotid artery were excluded from analysis of lesion morphology.

Analysis of the ultrasound scan results on the basis of the degree of stenosis shows that, although the disease was more severe in those with events, the difference did not reach statistical significance ( $>0 \cdot 1)$ (Table 1). However, on comparison of the morphological pattern of the

Table 1 Degree of stenosis in the carotid arteries of patients who had subsequent vascular events and in those who remained event free throughout the follow up period

\begin{tabular}{lllllll}
\hline & Clear & $1-19 \%$ & $20-49 \%$ & $50-74 \%$ & $75-99 \%$ & $\begin{array}{l}\text { Total } \\
\text { occlusion }\end{array}$ \\
\hline Vascular event group $(\mathrm{n}=37)$ & $19 \%(7)$ & $3 \%(1)$ & $22 \%(8)$ & $4 \%(11)$ & $32 \%(12)$ & $13 \%(5)$ \\
Event free group $(\mathbf{n}=90)$ & $27 \%(24)$ & $14 \cdot 5 \%(13)$ & $23 \%(21)$ & $14 \cdot 5 \%(13)$ & $18 \%(16)$ & $3 \%(3)$ \\
\hline
\end{tabular}

There was no significant difference found $(\mathrm{p}>0 \cdot 1)$ in the severity of the stenosis in the lesions between the groups.

Table 2 Ultrasound morphology of lesions, excluding total occlusion, in patients with carotid artery disease who had subsequent vascular events and in those who remained event free throughout the follow up period

\begin{tabular}{llll}
\hline & Homogeneous & Simple heterogeneous & $\begin{array}{l}\text { Complex } \\
\text { heterogeneous }\end{array}$ \\
\hline Vascular event group $(\mathrm{n}=25)$ & 0 & $16 \%(4)$ & $84 \%(21)$ \\
Event free group $(\mathrm{n}=63)$ & $22 \%(14)$ & $22 \%(14)$ & $56 \%(35)$ \\
\hline
\end{tabular}

There was a significant difference $(p<0.05)$ found in the distribution of homogeneous/simple heterogeneous lesions and complex heterogeneous lesions between the groups, with the patients who subsequently suffered a vascular event having a greater number of complex heterogeneous lesions. lesions, there was a statistically significant difference $(p<0.05)$ between the complex heterogeneous lesions and the combination of homogeneous/simple heterogeneous lesions. The patients who subsequently suffered events had a higher incidence of complex heterogeneous lesions (Table 2).

Carotid endarterectomy was performed on the symptomatic artery of 17 patients, of whom three suffered perioperative strokes. Discounting these perioperative event patients, the remaining postendarterectomy patients constituted $11 \%$ of non-event and $11 \%$ of subsequent event groups.

Antiplatelet therapy was administered to $73 \%$ of patients in the event group and $67 \%$ in the non-event group.

\section{Discussion}

Carotid endarterectomy is frequently performed on patients who present with retinal ischaemia. However, poor patient outcome has been reported in many studies, and patient selection is recognised as a major factor influencing long term outcome. ${ }^{58}$

The degree of stenosis caused by a carotid lesion has been critical in selecting those patients to whom surgery is offered. However, it has been found that retinal ischaemic symptoms are related to the morphological appearance of the lesion, even when the lesion causes lesser degrees of stenosis. ${ }^{124}$

Where the morphology of the lesion has been used in selecting patients for surgery, excellent results have been reported. ${ }^{2}$ However, a long term study of the outcome of non-surgically treated patients, whose carotid arteries have been morphologically assessed, has not hitherto been carried out.

In this study, 144 patients who presented with symptoms of retinal ischaemia were successfully followed for 2-7 years (mean 3.3 years). Cerebrovascular, cardiovascular, or retinal ischaemic symptoms subsequently developed in $26 \%$ of the group, $10 \%$ suffering stroke. A similar figure has been reported by other authors, ${ }^{35910}$ but the importance of the morphology of carotid lesions was not assessed in these studies.

Several studies have reported a relationship between vascular events and the severity of the stenosis in the carotid arteries. ${ }^{4-7}$ Our results of duplex ultrasound examination showed that although the percentage stenosis caused by the carotid disease in the patients who subsequently suffered events was more severe than in those patients who remained event free, the difference did not reach statistical significance $(p>0 \cdot 1)$. The morphology of lesions, and not the degree of stenosis, correlated with ischaemic symptoms and computed tomography evidence of infarction in a study of patients with symptomatic carotid disease." Although complex heterogeneous structures had been found to predominate in those lesions causing more than $50 \%$ stenosis, they are also found in symptomatic lesions causing $<50 \%$ stenosis. ${ }^{1}$.

Complex heterogeneous plaques have an uneven distribution of low level and high level ultrasound echoes within the lesion, and also 
contain low echo pools or have an irregular or pitted surface interfacing with blood flow. These lesions have been associated with episodes of both cerebral and retinal ischaemia. ${ }^{1212-15}$ However, the importance of morphological appearance in the prognosis of patients presenting with retinal ischaemia has not been evaluated previously.

A significant difference $(\mathrm{p}<0.05)$ in lesion morphology between the groups has been found. There was a greater incidence of complex heterogeneous plaques compared with the number of simple heterogeneous and homogeneous plaques in the subsequent event group of patients.

The vascular events occurred throughout the follow up period, with events being recorded from less than 1 year after initial presentation to 6 years. All of the non-fatal myocardial infarctions occurred in the second year of follow up. It has been reported that the maximum risk for stroke following cerebral transient ischaemic accidents is in the first year after the initial symptoms. ${ }^{16}$

There was a high incidence of cardiac events reported in the follow up; 17 (12\%) patients suffered myocardial infarction, seven being fatal. Other authors have found a similar incidence of cardiac events. ${ }^{6}{ }^{17} \mathrm{~A}$ relationship between the extent of vascular disease at different sites has been reported. ${ }^{6}{ }^{17-19}$ This study suggests that the finding of a complex heterogeneous plaque in the carotid system is indicative of risk in the coronary arteries, and that morphology may be more important than the size of the lesion, as complex heterogeneous lesions, irrespective of their size, were found to be associated with subsequent cardiovascular events.

Two patients who initially presented with amaurosis fugax reported further attacks, and one patient who initially presented with symptomatic retinal embolus, reported sudden tunnel vision. This was diagnosed as being due to a new retinal embolus. A further three patients reported non-vascular ophthalmic events.

Carotid endarterectomy has been advocated as a treatment of choice in patients presenting with retinal ischaemia and having a carotid stenosis of $>50 \% .^{2021}$ Both long and short term favourable results have been reported. ${ }^{2021}$ However, the advisability of this treatment has been questioned, and particularly the selection of patient to whom surgery is offered..$^{610}$ The results of more recent studies on carotid endarterectomy show significant benefit for symptomatic patients with a carotid stenosis of $70-99 \%$, and this treatment is not advocated in patients with less than $30 \%$ stenosis. $^{22}{ }^{23}$ However, in patients with stenosis of $30-69 \%$, the results are as yet unavailable. Selection of patients in these trials was based on the angiographic diagnosis of carotid artery stenosis. Unfortunately, contrast angiography does not provide information on plaque morphology. The ultrasound morphological appearance of carotid artery lesions in this investigation has been shown to be significant in the long term prognosis of patients with greater than $20 \%$ stenosis.

Carotid endarterectomy was performed on 17 patients, with surgical selection based primarily on the severity of stenosis. Excluding the cases of perioperative cerebrovascular accidents, endarterectomy was performed on $11 \%$ of patients in both event and non-event groups. This equal distribution suggests that selection criteria for treatment could be improved by including morphological appearance of the lesions.

The administration of antiplatelet therapy was found to be almost equally divided between the two groups, with no demonstrable effect being observed. Complex heterogeneous plaques have been shown in this study to have a high association with clinical symptoms and, therefore, it is possible that if patients had been selected for antiplatelet therapy on the basis of lesion morphology, a more accurate evaluation of the value of antiplatelet therapy might have been possible.

This study showed that in a group of 165 patients who presented with retinal ischaemia, there was a low incidence of further ophthalmic events, without a single incidence of subsequent blindness. However, $26 \%$ of the group had cerebrovascular or cardiovascular events in the follow up period. Complex heterogeneous lesions have been shown to be associated with symptoms of retinal ischaemia, both when the lesion has severe stenosis and when the lesion is of lesser significance in terms of size. ${ }^{12}$ In this study plaque morphology has been shown to have a significant association with long term prognosis, patients having complex heterogeneous plaques in their carotid vessels, irrespective of the degree of stenosis, having a $26 \%$ incidence of further cerebrovascular, cardiovascular, or retinal ischaemic events. This emphasises the importance of the role of the ophthalmologist in the detection of a significant number of patients potentially at risk of severe cerebrovascular or cardiovascular events.

1 O'Farrell CM, FitzGerald DE. Ultrasound morphology of carotid lesions in retinal ischaemia. Br $\mathcal{F}$ Ophthalmol 1992 76: 656-9.

2 Langsfield $\dot{M}$, Lusby RJ. Amaurosis fugax: the importance of carotid plaque morphology. Aust NZ $\mathcal{O}$ Ophthalmol 1988; 16: 275-80.

3 Chawluk JB, Kushner MJ, Bank WJ, Silver FL, Jamieson DJ, Bosley TM, et al. Atheromatous carotid artery disease in patients with retinal ischemic syndromes. Neurology 1988 ; 38: 858-63.

4 Torem S, Rossman ME, Schneider PA, Otis SM, Dilley RB, Bernstein EF. The natural history of amaurosis fugax with Bernstein EF. The natural history of amaurosis fugax with minor degrees of

5 Parkin PJ, Kendall BE, Marshall J, McDonald WI. Amaurosis fugax: some aspects of management. $\mathcal{F}$ Neurol Neurosurg Psychiatry 1982; 45: 1-6.

6 Hankey GJ, Slatter JM, Warlow GP. Prognosis and prognostic factors of retinal infarction: a prospective cohort study. BMF 1991; 302: 499-504.

7 Kolleratis CR, Lubow M, Hissong S. Retinal strokes 1. Incidence of carotid atheroma. $\mathcal{F A M} A$ 1972; 222: 1273-5.

8 Winslow CM, Solomon DH, Chassin MR, Kaescoff J, Merrick MJ, Brook RH. The appropriateness of carotid endarterectomy. $N$ Engl $\mathcal{M}$ Med 1988; 318: 721-7.

9 Marshall J, Meadows S. The natural history of amaurosis fugax. Brain 1968; 91:419-34.

10 Schwarcz TO, Eton D, Ellenby M, Stelmack T, McMahon T, Mulder S, et al. Hollenhorst plaques: retinal manifestations and the role of carotid endarterectomy. F Vasc Surg 1990 11: $635-41$.

11 Leahy AL, McCollum PT, Feeley TM, Sugrue M, Grouden MC, O'Connell DJ, et al. Duplex ultrasonography and selection of patients for carotid endarterectomy: plaque morphology or luminal narrowing? $\mathcal{f}$ Vasc Surg 1988; 8: 558-62.

12 Gray-Weale AC, Graham JC, Burnett JR, Byrne K, Lusby RJ. Carotid artery atheroma: comparison of pre-operative $B$ mode ultrasound appearance with carotid endarterectomy specimen pathology. $\mathcal{F}$ Cardiovasc Surg 1988; 29: 676-81.

13 Sterpetti AV, Schultz RD, Feldhaus RJ, Davenport KJ, Richardson M, Farina C, et al. Ultrasonographic features of carotid plaque and the risk of subsequent neurologic deficits. Surgery 1988; 104: 652-60. 
14 Davenport KL, Sterpetti AV, Schultz RD, Feldhaus RJ, Richardson M. Carotid artery plaque imaging and the risk of subsequent stroke. F Vasc Tech 1988; Oct.

15 O'Holleran LW, Kennelly MM, McClurken M, Johnson JM. Natural history of asymptomatic carotid plaque. Five year follow-up study. Am $₹$ Surg 1987; 154: 659-62.

16 Heyman A, Leviton A, Millikan CH. Transient focal cerebral ischemia: epidemiological and clinical aspects. Stroke 1974; 5: 227-83.

17 Poole CJM, Ross Russell RW. Mortality and stroke after amaurosis fugax. F Neurol Neurosurg Psychiatry 1985; 48: $902-5$.

18 Pfaffenbach DD, Hollenhorst RW. Morbidity and survivorship of patients with embolic cholesterol crystals in the
ocular fundus. Am $\mathcal{F}$ Ophthalmol 1973; 75: 66-72.

19 Mitchell JRA, Schwartz CJ. The relationship between arterial disease at different sites. Arterial disease. Oxford: Blackwell, 1965: 69-72.

20 Bernstein EF, Dilley RB. Late results following carotid endarterectomy for amaurosis fugax. $\mathcal{F}$ Vasc Surg 1987; 6 : 333-40.

21 Ehrenfeld WJ, Hoyt WF, Wylie EJ. Embolisation and transient blindness from carotid atheroma. Arch Surg 1966; 93: 787-94.

22 North American Symptomatic Carotid Endarterectomy Tria Collaborators. Beneficial effect of carotid endarterectomy in symptomatic patients with high-grade carotid stenosis. NEnglf Med 1991; 325: 449-53.

23 European Carotid Surgery Trialists Collaborative Group. MRC European Carotid Surgery Trial: interim results for symptomatic patients with severe $(70-99 \%)$ or with mild symptomatic patients with severe $(70-99 \%)$ or with
$(0-29 \%)$ carotid stenosis. Lancet 1991; 337: 1235-43. 